\title{
GMR
}

\section{Identification of a novel mutation of the EDA gene in $X$-linked hypohidrotic ectodermal dysplasia}

\author{
J.J. Xue ${ }^{1,2}$, B. Tan ${ }^{1}$, Q.P. Gao ${ }^{3}$, G.S. Zhu' ${ }^{1}$, D.S. Liang ${ }^{1}$ and L.Q. Wu ${ }^{1}$ \\ ${ }^{1}$ State Key Laboratory of Medical Genetics, Central South University, \\ Changsha, Hunan, China \\ ${ }^{2}$ Children's Hospital of Shanxi, Women Health Center of Shanxi, Taiyuan, \\ Shanxi, China \\ ${ }^{3}$ Department of Stomatology, Xiangya Hospital, Central South University, \\ Changsha, Hunan, China \\ Corresponding author: L.Q. Wu \\ E-mail: wulingqian@sklmg.edu.cn \\ Genet. Mol. Res. 14 (4): 15779-15782 (2015) \\ Received August 19, 2015 \\ Accepted October 14, 2015 \\ Published December 1, 2015 \\ DOI http://dx.doi.org/10.4238/2015.December.1.29
}

ABSTRACT. This study aimed to identify the disease-causing mutation in the ectodysplasin $A(E D A)$ gene in a Chinese family affected by X-linked hypohidrotic ectodermal dysplasia (XLHED). A family clinically diagnosed with XLHED was investigated. For mutation analysis, the coding region of EDA of 2 patients and 7 unaffected members of the family was sequenced. The detected mutation in EDA was investigated in 120 normal controls. A missense mutation (c.878T $>\mathrm{G}$ ) in $E D A$ was detected in 2 patients and 3 female carriers, but not in 4 unaffected members of the family. The mutation was not found in the 120 healthy controls and has not been reported previously. Our findings indicate that a novel mutation (c.878T $>\mathrm{G}$ ) of EDA is associated with XLHED and adds to the repertoire of EDA mutations.

Key words: c.878T>G mutation; Ectodysplasin gene; Novel mutation; $\mathrm{X}$-linked hypohidrotic ectodermal dysplasia 


\section{INTRODUCTION}

X-linked hypohidrotic ectodermal dysplasia (XLHED) is a rare hereditary disease and occurs in less than 1 in 100,000 births (Zonana, 1993). The condition is characterized by symptoms including hypohidrosis, hypodontia and sparse hair, abnormal development of nails, immunodeficiency, and characteristic faces with juga forehead and chin, saddle nose, low-set ears, maxillary hypoplasia, thick and prominent lips, wrinkles, and pigment sedimentation around the orbit of the eyes. According to previous reports, XLHED is X-linked recessive or X-linked intermediate. Hemizygous male patients are often more severely affected, while heterozygous females show normal or moderate features (Itin and Fistarol, 2004). XLHED is known to be caused by mutations in the ectodysplasin $\mathrm{A}(E D A)$ gene, which is located at Xq12-q13.1. EDA encodes a protein of 391 amino acids and is comprised of an intracellular domain, a transmembrane domain, a furin subdomain, a 19 repeat Gly-X-Y collagenous domain, a tumor necrosis factor (TNF) homology subdomain, and a cysteine-rich C-terminal domain (Liu et al., 2012). EDA is a trimeric type II transmembrane protein that can release the trimer TNF core domain, which can then bind its receptor ectodysplasin-A receptor (EDAR) (Chen et al., 2001). A previous study demonstrated that the TNF homology domain is crucial for the structure and function of the EDA protein (Song et al., 2009). This domain is located at the $\mathrm{C}$-terminal end of the protein and is composed of 10 predicted antiparallel $\beta$-sheets, which are necessary for the binding of EDA to EDAR (Ezer et al., 1999). The recognition and interaction between EDA and EDAR may activate the nuclear factor- $\kappa \mathrm{B}$ and $\mathrm{c}-\mathrm{Jun}$ $\mathrm{N}$-terminal kinase pathways involved in the regulation of ectodermal morphogenesis (Huang et al., 2006). Here, we describe the clinical features and molecular characterization of a novel point mutation in the EDA gene in a Chinese family with XLHED (Figure 1).

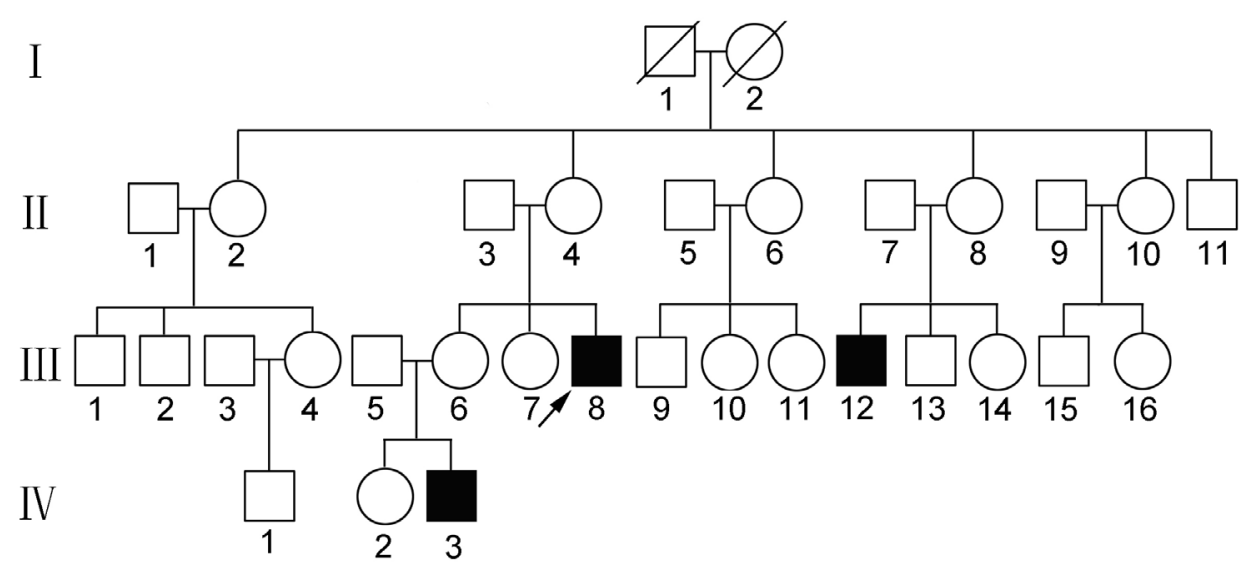

Figure 1. Pedigree of the reported family with XLHED.

\section{MATERIAL AND METHODS}

The proband (III8) was a 21-year-old man. His appearance displayed the characteristic features of hypohidrotic ectodermal dysplasia (HED): sparse hair, sparse eyebrows, thin and dry skin, and specific facial features, i.e., frontal bossing, saddle nose, prominent lips, juga chin, 
maxillary hypoplasia (Figure 2A). He had only 2 permanent central incisors in the maxilla. However, no abnormalities in his finger or toenails were observed. He had a father, mother, and 2 sisters, all of whom were healthy. Pedigree analysis showed that 3 males in 2 successive generations of the family were affected, indicating that the disease may have an X-linked recessive pattern in this family.

Genomic DNA was extracted from peripheral blood leukocytes of all available family members (II2, II6, II8, II10, II1, III6, III7, III8, and IV3) using standard protocols. All coding regions and the intron/exon boundaries of EDA were amplified by polymerase chain reaction, and the amplification products were directly sequenced using the $\mathrm{ABI}$ Big Dye terminator cycle sequencing kit (Applied Biosystems, Foster City, CA, USA) according to the manufacturer instruction. Sequences were determined using an ABI 3100 sequencer (Applied Biosystems).

\section{RESULTS}

Mutation analysis revealed that 2 patients (III8, IV3) were hemizygous for a novel missense mutation c.878T>G (p.Leu293Arg) in exon 8 of EDA (Figure 2B). The mutation was present in the females of the family (II6, II8, and III6), and these females were heterozygous c.878T>G mutation carriers. The mutation was absent in II2, II10, II11, and III7. The patients were hemizygous for this genotype and the unaffected male member was normal, indicating that the mutation cosegregates with HED in the family.

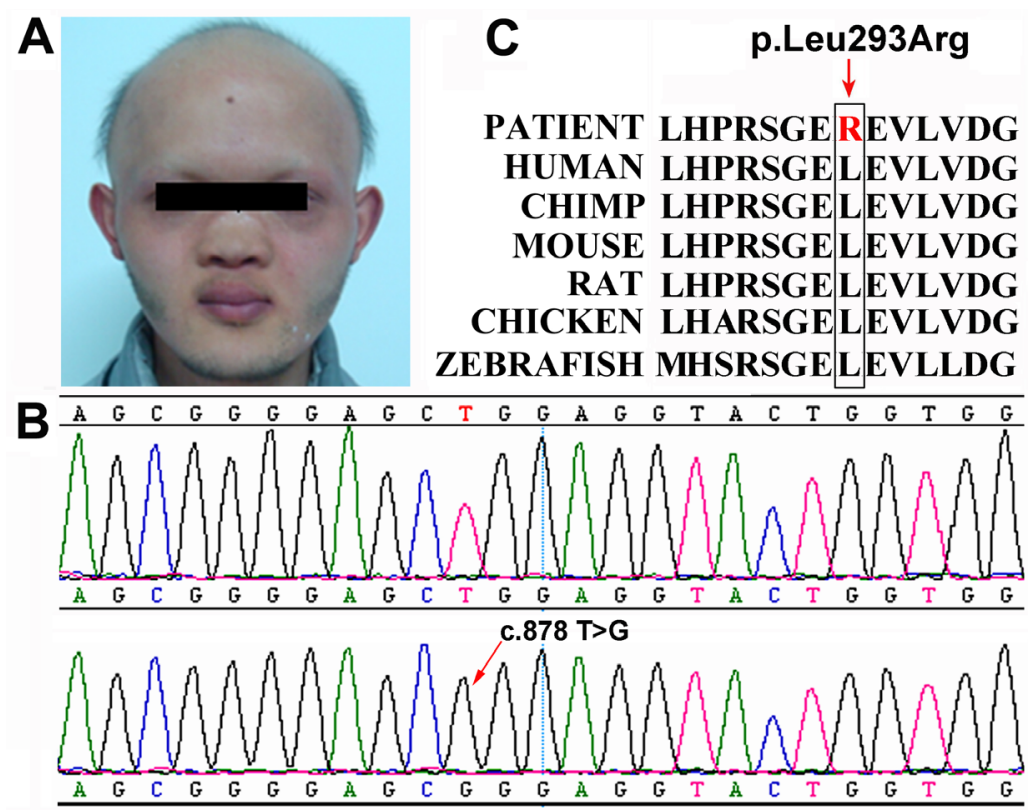

Figure 2. A. Photograph showing the hypohidrotic ectodermal dysplasia phenotypic characteristics of the proband. B. Sequence analysis of EDA in the healthy control (top) and the proband (bottom). C. Evolutionary conservation of amino acid affected by the mutation. The mutant allele is boxed.

The missense mutation has not been reported previously and was not found in 120 healthy controls. The mutation results in substitution of leucine 293 with arginine, and the leucine at this position is conserved (Figure $2 \mathrm{C}$ ), suggesting that it is functionally important. 


\section{DISCUSSION}

Over 100 mutations of EDA have been reported to be associated with HED, including missense, small deletion, insertion, nonsense, and splice-site mutations, as well as complete gene deletion. More than $60 \%$ of point mutations are found in exons 7,8 , and 9 , which encode for the TNF homology subdomain. In this study, we identified a novel missense mutation (c.878T $>\mathrm{G}$ ) located in the TNF homology subdomain of the EDA protein. A missense mutation (c.878T $>C$ ) at the same codon has been reported to lead to HED (Cluzeau et al., 2011), suggesting that leucine 293 may play a key role in the function of the TNF-like domain and the EDA protein.

\section{Conflicts of interest}

The authors declare no conflict of interest.

\section{ACKNOWLEDGMENTS}

We thank the participating family members for their help and support. Research supported by grant from the National Key Technology R\&D Program of China (\#2012BAI09B05) and the National Natural Science Foundation of China (\#81200875).

\section{REFERENCES}

Chen Y, Molloy SS, Thomas L, Gambee J, et al. (2001). Mutations within a furin consensus sequence block proteolytic release of ectodysplasin-A and cause X-linked hypohidrotic ectodermal dysplasia. Proc. Natl. Acad. Sci. U. S. A. 98: 7218-7223.

Cluzeau C, Hadj-Rabia S, Jambou M, Mansour S, et al. (2011). Only four genes (EDA1, EDAR, EDARADD, and WNT10A) account for $90 \%$ of hypohidrotic/anhidrotic ectodermal dysplasia cases. Hum. Mutat. 32: 70-72.

Ezer S, Bayés M, Elomaa O, Schlessinger D, et al. (1999). Ectodysplasin is a collagenous trimeric type II membrane protein with a tumor necrosis factor-like domain and co-localizes with cytoskeletal structures at lateral and apical surfaces of cells. Hum. Mol. Genet. 8: 2079-2086.

Huang C, Yang Q, Ke T, Wang H, et al. (2006). A novel de novo frame-shift mutation of the EDA gene in a Chinese Han family with hypohidrotic ectodermal dysplasia. J. Hum. Genet. 51: 1133-1137.

Itin PH and Fistarol SK (2004). Ectodermal dysplasias. Am. J. Med. Genet. C Semin. Med. Genet. 131: 45-51.

Liu Y, Yu X, Wang L, Li C, et al. (2012). Mutation p.Leu354Pro in EDA causes severe hypohidrotic ectodermal dysplasia in a Chinese family. Gene 491: 246-250.

Song S, Han D, Qu H, Gong Y, et al. (2009). EDA gene mutations underlie non-syndromic oligodontia. J. Dent. Res. 88: $126-131$. Zonana J (1993). Hypohidrotic (anhidrotic) ectodermal dysplasia: molecular genetic research and its clinical applications. Semin. Dermatol. 12: 241-246. 